\title{
Linx
}

Revue des linguistes de l'université Paris X Nanterre

47 | 2002

Du sens au sens

\section{Les bases de la quantification réciproque}

On foundations of reciprocical quantification

\section{Francis Renaud}

\section{OpenEdition}

Journals

Édition électronique

URL : http://journals.openedition.org/linx/586

DOI : $10.4000 /$ linx.586

ISSN : 2118-9692

\section{Éditeur}

Presses universitaires de Paris Nanterre

\section{Édition imprimée}

Date de publication : 1 décembre 2002

Pagination : 89-106

ISSN : 0246-8743

\section{Référence électronique}

Francis Renaud, «Les bases de la quantification réciproque », Linx [En ligne], 47 | 2002, mis en ligne le 01 juin 2003, consulté le 19 avril 2019. URL : http://journals.openedition.org/linx/586 ; DOI : 10.4000/ $\operatorname{lin} x .586$

Département de Sciences du langage, Université Paris Ouest 


\title{
Les bases de la quantification réciproque
}

\author{
Francis Renaud, EHESS, CRLAO
}

En hommage aux talents multiples de Michel Galmiche.

\begin{abstract}
Donner une représentation logique des constructions réciproques s'avère assez aisé, surtout si on travaille au coup par coup (voir des exemples en annexe). Mais cette limpidité apparente des interprétations cache le problème redoutable de trouver une valeur générale (glose ou formule) valable pour tous les cas

Dalrymple et al. (1998) ont imaginé une solution astucieuse à cette difficulté. Ils supposent que le contexte est toujours en mesure d'éliminer un certain nombre de valeurs et qu'il suffit alors de garder l'interprétation restante la plus forte. En parcourant leurs exemples, on s'aperçoit rapidement qu'ils font jouer un rôle décisif au contexte, sans nous donner les moyens d'en extraire l'information utile. Au point que dans certains cas, le contexte semble jouer un rôle bien plus important que le contenu de la phrase elle-même !

Face à cette difficulté, il nous a semblé bon d'entreprendre une étude systématique de toutes les structures donnant une interprétation réciproque et d'essayer de définir des tests précis permettant de les classer d'une manière relativement homogène. Ce travail devrait nous permettre plus tard de définir précisément les paramètres de contrôle de l'interprétation réciproque. Nous nous sommes efforcé de trouver, par tâtonnement, des tests syntaxico-sémantiques permettant d'organiser l'ensemble des constructions réciproques en classes relativement homogènes.

Nous tenterons d'analyser la sous-classe des constructions réciproques définies par une relation entre un domaine et un prédicat à deux arguments référentiels (une relation binaire). Cette classe est dotée de propriétés quantificationnelles très spéciales :

- ni distributives (propriétés liées en général à la quantification généralisée, relation entre deux ensembles d'individus - un domaine et l'ensemble des référents liés à un argument individuel d'un prédicat)
\end{abstract}


- ni collectives, liées à une relation entre un domaine et l'ensemble des parties des référents liés à un argument pluriréférent d'un prédicat mais quantification de type $<1,2>$ (au sens de Westerstahl 1994, comme nous verrons).

\section{Conditions d'existence de la construction réciproque}

L'emploi des formes un N..., un autre... dans des GN disjoints permet de puiser des éléments distincts de la classe $\mathrm{N}$ :

(1) Une personne peut être sensibilisée par un allergène et rester asymptomatique et une autre peut devenir allergique très rapidement

Ces éléments distincts peuvent par ailleurs être rassemblés dans une classe unique pour fournir le contenu référentiel d'un $\mathrm{GN}$ :

(2) Dans ce centre, les uns utilisent des macs, les autres des pc. Mais les uns et les autres ont des problèmes de stabilité du système

L'expression les uns et les autres permet de rassembler deux ensembles référentiels disjoints dans la même classe. Qu'en est-il lorsqu'on recrute ces ensembles dans la même proposition :

(3) Souvent, chez les politiques, les uns accusent les autres de tricher

(4) Les politiques s'accusent souvent les uns les autres de tricher

Dans cette dernière phrase, le GN les politiques, en combinaison avec les marqueurs se... les uns les autres, fait plus que rassembler un ensemble d'individus distincts dans une même classe : il rompt la dissymétrie figée de (3) entre accusateurs et accusés, chacun pouvant être accusateur ou accusé dans (4). Le clitique se, marqueur d'identité référentielle, distribue les événements sur le domaine : un politique accuse un politique (lui-même ou un autre), la locution les uns les autres, contraint la distribution à opérer sur des individus différents. On voit donc que la construction réciproque basée sur la structure $\mathrm{GN}_{1} \mathrm{~V} \quad \mathrm{GN}_{2}$, se caractérise en première approximation par deux procédures :

- une construction d'une classe homogène de plusieurs individus, pouvant être à tour de rôle agents et patients, mais tous référencés en position sujet $\mathrm{GN}_{1}$

- une quantification sur des couples d'individus différents, marquée par la locution pronominale les uns les autres, en position objet $\mathrm{GN}_{2}$.

La construction réciproque s'appuie donc à la fois sur la disjonction référentielle intrapropositionnelle et sur la construction d'une classe homogène permettant d'effectuer une quantification très particulière entre des référents jouant des rôles interchangeables dans leur interaction.

Voyons d'abord de manière plus précise le mode de dérivation des constructions réciproques à l'intérieur de la proposition. Il s'agit d'un procédé de construction simple, régulier et productif des structures réciproques.

Prenons le prédicat être en concurrence : 
(5) Un individu peut être en concurrence avec un autre individu

(6) Un procédé de craquage du méthane peut être en concurrence avec un autre procédé de craquage

En faisant parcourir aux deux GN une classe homogène, on peut obtenir la forme générale :

(7) Ces individus sont en concurrence

(8) Ces procédés de craquage du méthane sont en concurrence

Par contre un individu ne peut pas être en concurrence avec un procédé de craquage du méthane et on ne peut avoir

(9) \#ces individus et ces procédés de craquage sont en concurrence

Ce procédé de construction d'une classe homogène s'appuie sur le procédé de fusion référentielle (Schnedecker, Bianco 2000) d'extensions disjointes :

(10) Max a offert un cadeau à Fabienne. (L'un et l'autre/Tous les deux) sont ravis Ils se sont offert des cadeaux l'un à l'autre

(Pour être naturelle, la forme forte, avec la locution l'un à l'autre, doit être dans un contexte introduisant une insistance sur la réciprocité).

Lorsqu'on a, comme ici, deux monoréférents disjoints, la reprise pronominale se contente de fusionner les extensions des deux $G N:\{\max \} \cup\{f a b\}=\{\max , f a b\}$. Mais pour analyser une classe plus grande telle que :

(11) Dans la famille, pour les fêtes, nous nous offrons des cadeaux

il faut cumuler itérativement les fusions référentielles de tous les $\mathrm{x}$, y qui parcourent la classe des membres de la famille et tels que x offre un cadeau à y. En fait l'opération ne peut être décrite correctement qu'en utilisant une représentation sémantique. Nous nous contenterons dans un premier temps de cette approche de surface intuitive.

Pour pouvoir dériver une construction réciproque sur les arguments $\mathrm{x}$ et $\mathrm{y}$ d'un prédicat P.x.y il faut donc construire une classe homogène d'individus par « l'accumulation itérative » des deux arguments parcourant les extensions du prédicat. La classe une fois constituée peut être quantifiée librement par un Det $\mathrm{N}_{\text {plur }}$ pluriréférent. La construction réciproque permet le parcours de l'extension de ce $\mathrm{GN}_{\text {plur }}$ par paires $(\mathrm{x}, \mathrm{y})$ d'individus distincts :

- s'il n'y a pas d'extension commune entre les deux arguments $\mathrm{x}, \mathrm{y}$ :

(12) Max a rogné (les pages du livre/* son copain)

(13) *(les copains/les pages/Max et les pages) se sont rognés

il n'y a pas de construction réciproque possible

- si une intersection des extensions est possible :

(14) Max a loupé de peu (son train/son ami) à la gare 
la construction réciproque se fera uniquement dans l'intersection ${ }^{1}$ :

(15) *Max et son train se sont loupés de peu

(16) (Max et son ami/les amis) se sont loupés de peu à la gare

- un prédicat unaire ne peut pas donner d’interprétation réciproque

(17) *Ils baillaient les uns les autres

sauf si on le transforme par un factitif en un prédicat complexe à deux arguments :

(18) i fait bailler $\mathrm{j} \mapsto$ Ils se font bailler (les uns les autres)

$(\mapsto$ se lit « dériver par fusion itérative »)

- les deux référents $\mathrm{x}$, y peuvent être pris dans les extensions du sujet et d'un complément (direct ou indirect, voir ex. précédents), mais aussi d'un sujet et d'un complément adnominal :

(19) Max serra la main de Paul $\mapsto$ Ils se sont serré la main

Les locatifs peuvent aussi intervenir. On peut avoir aussi bien l'intersection d'un sujet et d'un locatif :

(20) cette orbitale atomique s'emboîte dans celle-là

les orbitales atomiques s'emboîtent (les unes dans les autres) à la manière des pelures d'oignon

que celle d'un objet et d'un locatif :

(21) Max a mis un sac dans un autre sac

Max a mis les sacs les uns dans les autres

- la contrainte de disjonction référentielle $\mathrm{x} \neq \mathrm{y}$ se vérifie (négativement) avec un prédicat attributif comme la copule qui précisément assure une identité référentielle non temporelle (Renaud, 1999) :

(22) Le chef de l'Etat est le chef des Armées

* Ils sont l'un l'autre (Il est l'un et l'autre n'a pas de valeur réciproque)

- les deux référents $\mathrm{x}$ et y peuvent jouer deux rôles sémantiques aussi bien opposés que semblables :

Dans les rôles d'agent / patient, on a :

(23) Max critique Paul (\# Paul critique Max) $\mapsto$ ils se critiquent

Avec un verbe symétrique, les rôles sont semblables :

(24) L'étude clinique anglaise s'accorde avec la française à démontrer que la maladie de Hodgkin est extrêmement chimiosensible ( $\leftrightarrow$ l'étude clinique

1 intersection du domaine et du codomaine du prédicat binaire P. Le domaine de $\mathrm{P}$ est l'ensemble des référents pouvant satisfaire le premier argument $\mathrm{x}$ de $\mathrm{P}: \operatorname{dom} . \mathrm{P}=\lambda \mathrm{x} \exists \mathrm{y}$ P.x.y et le codomaine l'ensemble des référents pouvant satisfaire le deuxième argument y $: \operatorname{cod} . \mathrm{P}=\lambda \mathrm{y} \exists \mathrm{x}$ P.x.y. 
française s'accorde avec l'anglaise à démontrer que...)

$\mapsto$ les études cliniques s'accordent à démontrer que...

Le mode de construction des structures symétriques est donc semblable à celui des structures réciproques.

Sur la base de l'analyse précédente, nous pouvons avancer une première hypothèse sur la représentation sémantique de la construction réciproque. L'idée que

(25) Les passagers se bousculèrent (les uns les autres) pour entrer dans le wagon

s'analyse à partir d'un parcours de couples d'individus $\mathrm{x}$, y distincts, du domaine de quantification (les passagers, en situation) et s'appliquant à la propriété «x bouscule y » peut se représenter par une relation liant le domaine à cette propriété binaire :

RECIP.passager. $(\lambda x \lambda y$ $\exists$ e bousculer.e.x.y)

Si on comprend (25) comme «pour tout passager $\mathrm{x}$, il en existe un autre $\mathrm{y}$, tel que $\mathrm{x}$ bouscule ou est bousculé par y », on prendra alors

$$
\text { RECIP.X.P }=|\mathrm{X}| \geq 2 \wedge \forall \mathrm{x}(\mathrm{X} . \mathrm{x} \rightarrow \exists \mathrm{y}(\mathrm{X} . \mathrm{y} \wedge \mathrm{x} \neq \mathrm{y} \wedge(P .\{\mathrm{x}\} .\{\mathrm{y}\} \vee P .\{\mathrm{y}\} .\{\mathrm{x}\})))
$$

L'opérateur RECIP est un quantificateur de type $<1,2>$ au sens de Westerstahl (1994), c'est-à-dire, qu'il s'interprète dans une structure ayant pour univers $\mathrm{U}$, comme une relation entre une partie de $\mathrm{U}$ (le domaine) et une partie de U x U (la propriété binaire) :

$$
\mathrm{RECIP} \subseteq \wp(\mathrm{U}) \times \wp(\mathrm{U} \times \mathrm{U}) \quad[\wp(\mathrm{U}) \text { ensemble des parties de l'ensemble } \mathrm{U}]
$$

Cette proposition a déjà été faite par Dalrymple et al. (1998) et Keenan (1987). Mais il est possible d'être encore plus précis. Nous avons vu que par construction même, le domaine $\mathrm{X}$ est formé par l'ensemble des éléments parcourus par les couples $(\mathrm{x}, \mathrm{y})$ tels que P.x.y. Le relation RECIP.X.P définit donc un objet mathématique bien connu : un graphe $\mathrm{G}=(\mathrm{X}, \mathrm{P})$ avec $\mathrm{X}$ l'ensemble des sommets et P l'ensemble des arcs. On pourra donc donner, au choix, une représentation algébrique ou géométrique de la réciprocité.

On pourrait se demander si le nombre d'événements liant deux individus intervient ou bien si seule l'existence d'au moins un événement est pertinente. En d'autre terme, doit-on étiqueter les arcs? (deux sommets peuvent-ils être liés par plusieurs arcs ?) la quantification porte-elle sur les individus et les événements ? Par exemple, un modèle possible de la phrase :

(26) [groupe de quatre amis] On s'est déjà rendu service

peut être

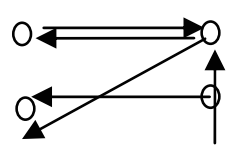

Par contre, si seuls deux amis du groupe se sont rendus service, peu importera le nombre de fois où ils l'ont fait, la structure suivante, avec les mêmes quatre individus, ne sera jamais un modèle : 
Francis Renaud
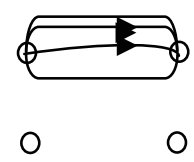

Il ne faut donc pas étiqueter les arcs par les événements : la quantification se limite aux individus.

Les quantificateurs événementiels peuvent cependant intervenir mais lorsqu'ils le font, ils opèrent indépendamment de la réciprocité (interprétation cumulative) :

(27) Ces deux dernières années, on s'est invités une dizaine de fois

Avec X le groupe des quatre amis, la représentation sera :

$\mid \lambda \mathrm{e} \exists \mathrm{x} \exists \mathrm{y} X . \mathrm{x} \wedge \mathrm{X} . \mathrm{y} \wedge \mathrm{x} \neq \mathrm{y} \wedge$ inviter.e.x.y $\wedge$ inter.e $\subseteq[1999,2000] \mid \approx 10$

$\wedge$ RECP.X. $(\lambda x \lambda y$ ye inviter.e.x.y $\wedge$ inter.e $\subseteq[1999,2000])$

- au critère sémantique esquissé jusque là, s'ajoute un critère structural : n'importe quelle paire de GN de la phrase simple ne peut être mobilisée par la procédure de construction. Ainsi

(28) La susceptibilité des uns suscite la risée des autres

(29) Vincent était littéralement subjugué par le récit des voyages de Gauguin

ne peuvent donner de construction réciproque :

(30) * la susceptibilité des ultranationalistes suscite la risée les uns des autres

(31) * ils étaient littéralement subjugués par le récit des voyages les uns des autres

Nous verrons dans la section 3 qu'il est toutefois possible de lister l'ensemble des constructions syntaxiques capables de fournir des constructions réciproques.

Nous avons vu jusque là comment le procédé de construction réciproque pouvait opérer à l'intérieur d'une proposition syntaxique simple. Que se passe-t-il lorsqu'on mobilise deux GN pris dans deux propositions, à temps fini, distinctes :

(32) Vincent était toujours inquiet quand Théo partait

$\leftrightarrow$ Ils étaient toujours inquiets quand (ils/l'un ou l'autre/* l'un l'autre) partaient

(33) Anne s'étonne que Paul plaise à Sylvie

$\leftrightarrow$ Elles s'étonnent que Paul (leur plaise/*plaise l'une à l'autre)

Les formes acceptables associées n'ont pas l'interprétation réciproque. Pour obtenir cette interprétation sur un domaine à deux éléments, on peut recourir à la tournure suivante :

(34) Chacun était inquiet quand l'autre partait

(35) Chacune s'étonne que Paul plaise à l'autre

Lorsque l'on passe à un domaine de quantification comportant beaucoup plus que deux éléments, on peut hésiter sur le choix de la glose : 
(36) Chacun était inquiet quand (un autre partait/(d'autres/les autres) partaient)

De plus, les difficultés s'accroissent encore, lorsque l'on sort du domaine de la phrase complexe (comme en 32-33) et qu'on mobilise des GN dans des phrases distinctes :

(37) Après avoir bu, Van Gogh avait un comportement inquiétant. Parfois, Gauguin s'éveillait la nuit et le trouvait debout à côté de lui, le regardant fixement, sans bouger

Bien qu'on puisse imaginer une situation du monde où les rôles des deux amis soient interchangés régulièrement, on a quelques difficultés à mettre en oeuvre le dernier procédé de construction réciproque :

(38) ?? Après avoir bu, chacun avait un comportement inquiétant. Parfois, l'autre s'éveillait la nuit et le trouvait debout à côté de lui, le regardant fixement, sans bouger

On voit donc qu'il n'y a pas de modèle standard de construction réciproque dans le domaine interphrastique.

Pour finir, signalons l'interférence du phénomène de réciprocité (à l'intérieur d'une proposition) avec l'anaphore sur le domaine de quantification réciproque :

(39a) Max pense que Paul le critique dans le dos

$\leftrightarrow$ b Les chercheurs pensent qu'ils se critiquent (les uns les autres) dans le dos

Il est difficile d'avoir des intuitions claires dans ce domaine mais il me semble que dans (39b) l'opérateur de réciprocité a la portée étroite (c'est-à-dire que «chacun pense que l'autre le critique» n'est pas un modèle). Heim, Lasnik \& May (1991) s'appuient uniquement sur ce genre de structures pour proposer une analyse générativiste de la réciprocité. Pour notre part, nous préférons commencer l'analyse de la réciprocité à l'intérieur d'une seule proposition avant d'envisager l'étude de son interférence avec le phénomène de l'anaphore interphrastique.

Pour terminer et résumer cette section, nous donnerons quelques définitions.

Notons GNi un groupe nominal monoréférent dont le référent est précisément i.

1) Si dans les phrases GNi $\mathrm{V} \quad \alpha \quad \mathrm{GNj} \quad \boldsymbol{\beta}$ les deux groupes nominaux monoréférents $\mathrm{GN}_{\mathrm{i}}$ et $\mathrm{GN}_{\mathrm{j}}$ disjoints $(\mathrm{i} \neq \mathrm{j}$ ) peuvent parcourir la même classe nominale $\mathrm{N}_{0}$ (décrite par le $\mathrm{GN}_{\text {pluri }}$ pluriréférent, tel que : les $\mathrm{N}_{0}$ )

2) et si la structures GNi $\mathrm{V} \quad \alpha$ GNj $\quad \beta$ appartient à un des schémas syntaxiques donnés dans la section 2 suivante, alors $G_{\text {pluri }}$ V' $\alpha$ Loc_R $R_{\text {Pr }}$ $\beta$ possède une valeur quantificationnelle réciproque de type $<1,2>$ (avec $V^{\prime}=$ se $V$ pour $\mathrm{GNj}$ accusatif ou datif et $V^{\prime}=\mathrm{V}$ pour les autres cas et Loc_R $R_{\operatorname{Pr}}$ les locutions de réciprocité, le tout explicité plus loin). Une règle semblable s'applique au cas du $\mathrm{GN}_{\mathrm{i}}$ en fonction objet.

Nous appellerons forme source la structure GNi V $\alpha \mathrm{GNj} \beta$ où les groupes nominaux $\mathrm{GNi}$ et $\mathrm{GNj}$ peuvent parcourir une classe nominale $\mathrm{N}_{0}$, et nous appellerons 
construction $\mathbf{R}$ (réfléchie-réciproque), la construction non distributive $\mathrm{GN}_{\text {pluri }} \mathrm{V}^{\prime} \alpha \beta$ associée, avec $\mathrm{GN}_{\text {pluri }}$ le groupe nominal pluriréférent de classe $\mathrm{N}_{0}$.

Nous notons Loc_R $R_{\operatorname{Pr}}$ les locutions de réciprocité telles que les uns Pr les autres, avec Pr une préposition. La construction réciproque $\mathrm{GN}_{\text {pluri }} \mathrm{V}^{\prime} \alpha \beta$ sera dite faible et celle comportant une locution de réciprocité $G_{\text {pluri }} V^{\prime} \alpha \quad L_{0} \_R_{\operatorname{Pr}} \beta$ sera dite forte. Les termes «faible» et «fort» s'emploient aussi à propos des interprétations, sans qu'il y ait la moindre correspondance entre ces valeurs syntaxiques et sémantiques.

L'ensemble des référents, membres de la classe homogène, sont appariés par couples de deux qui soit participent à une même action (en jouant éventuellement des rôles différents) soit sont liés par une relation psychologique, sociale ou informationnelle.

La contrainte sémantique 1) est suffisante sans être nécessaire, puisqu'il existe une classe restreinte mais significative de constructions symétriques non réductibles à un parcours de couples d'individus sur une classe :

(40a) Les élèves et les professeurs se sont réunis ( $\varepsilon /$ les uns avec les autres) pour réclamer plus de moyens

(41a) Les revenus, les impôts, l'amortissement et l'accumulation du capital s'ajoutent les uns aux autres pour donner le Revenu National

ne peuvent être reliées à une itération de phrases comme :

(40b) ?Max, un élève, s'est réuni avec un professeur pour réclamer plus de moyens

(41b) Les revenus s'ajoutent aux impôts pour donner le Revenu National

La phrase 40a, dans le contexte d'un lycée, laisse supposer qu'il n'y a eu qu'un seul événement "se réunir», alors que l'itération cumulative supposerait autant de réunions que de couples $(\mathrm{x}, \mathrm{y})$ parcourant le domaine (comme dans l'exemple 25 les passagers se bousculèrent, où on a une multiplicité d'événements élémentaires « $\mathrm{x}$ bouscule $\mathrm{y} »)$.

De même

(42a) Les étoiles de mer parsèment le fond du lagon de joyaux immobiles

bien que non distributive, ne peut être reliée à une itération de phrases comme :

(42b) *cette étoile de mer-ci parsème avec celle-là le fond du lagon de joyaux immobiles

Là aussi, on n'a qu'un seul état e satisfaisant parsemer.e.étoile, indécomposable en états élémentaires entre les actants (à la différence d'une phrase comme les documents sont posés les uns sur les autres (ex. 3 de l'Annexe), où on a une multiplicité d'états e "être posé»).

On a par contre des propriétés de symétrie : 
(43) Les étoiles de mer et les crinoïdes parsèment le fond du lagon de joyaux immobiles

(44) Les étoiles de mer parsèment avec les crinoïdes le fond du lagon...

Autres prédicats du même type: être aligné, s'annuler, s'allier, cohabiter, communiquer, être agrafé...

Cette sous-classe de constructions «réciproques" ne relève donc pas de la quantification de type $<1,2>$ mais d'interprétations collectives - l'éventualité globale est indécomposable en éventualités élémentaires.

Nous résumerons les constructions dans le tableau suivant :

NON DISTRI

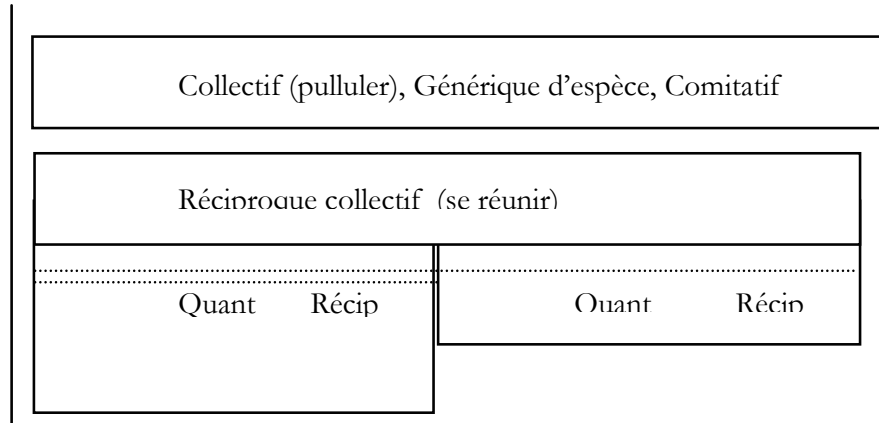

\section{Schémas syntaxiques productifs de la réciprocité}

Nous reprenons dans cette section l'étude de la quantification réciproque de type $<1,2>$. Nous indicerons le mot vide $\varepsilon_{\mathrm{R}}$ par $\mathrm{R}$, pour indiquer la construction réciproque faible. Nous distinguons trois grandes classes suivant que la forme faible est marquée, non marquée ou est absente.

\subsection{Constructions réciproques, avec formes faibles cliticisées}

Dans cette classe, la forme faible $\mathrm{GN}_{\text {pluri }}$ se $\mathrm{V}$ est marquée par le clitique se (à la $3^{\text {ième }}$ pers.). La forme forte est marquée par la locution Loc_R $R_{\operatorname{Pr}} \rightarrow$ mutuellement / les uns Pr les autres. Les formes faibles et fortes ont en général des valeurs différentes.

2.1.1. Verbe source transitif : GNi V GNj $\omega \rightarrow G_{\text {pluri }}$ se V $\left(\varepsilon_{R} /\right.$ Loc_R $\left.R_{\varepsilon}\right)$ $\omega$

Intersection du sujet et de l'objet direct. (i.e. intersection des extensions du sujet et de l'objet). Il s'agit d'exemples comme :

(1) un passager i bouscula un autre passager j pour entrer au plus vite dans le wagon Les passagers se bousculèrent $\left(\varepsilon_{\mathrm{R}} /\right.$ les uns les autres) pour entrer au plus vite dans le wagon

Après avoir testé toutes les tables de Guillet et Leclère (1992) nous pouvons affirmer que toutes les structures comportant un sujet et un objet direct sont 
susceptibles de donner une construction réciproque dans la mesure où la contrainte sémantique est satisfaite (existence d'une intersection homogène des extensions) et où les situations du monde correspondantes existent. Les néologismes transitifs donnent aussi des constructions réciproques. Les prédicats peuvent être dynamiques ou statifs (mal se supporter, s'aimer) mais à notre connaissance il n'y a pas d'adjectif transitif direct (quand les adjectifs ont un deuxième argument, il est introduit par une préposition, cf. 2.2).

Suivant les propriétés du prédicat, la forme $\mathrm{R}$ réciproque peut aussi posséder une valeur réfléchie. Cette valeur peut être privilégiée (ex. 2), fusionnée avec la réciprocité (ex. 3) ou interdite (ex. 4):

(2) J'ai abonné mon frère à La Recherche Nous nous sommes abonnés ( $\varepsilon_{\mathrm{R}}$ réfl ?récip/mutuellement) à La Recherche

(3) un invité i présenta un autre invité j à Fabienne

Les invités se présentèrent $\left(\varepsilon_{\mathrm{R}}\right.$ réfll $\cup$ récip/mutuellement) à Fabienne

(4) À la caisse, un client $\mathrm{i}$ suivait patiemment un autre client $\mathrm{j}$

À la caisse, les clients se suivaient patiemment ( $\varepsilon_{\mathrm{R}}$ récip/l'un l'autre)

Remarquons qu'en (3), la forme faible $\mathrm{R}$ n'a pas l'ambiguité réfléchie/réciproque puisque les deux valeurs peuvent coexister, certains invités se présentent eux-mêmes, d'autres sont présentés :

$\forall \mathrm{x}$ (invité. $\mathrm{x} \rightarrow \exists \mathrm{y}$ (invité.y $\wedge \exists$ e présenter.e.x.y.(à.fabienne)))

[y peut être identique ou différent de $\mathrm{x}$ ]

On a une seule représentation correspondant à une valeur monosémique.

Dans une phrase complexe à deux prédicats partageant un argument P.x.y, Q.x.z, la construction réciproque se construit autour du prédicat dont les arguments sont fusionnés même s'ils concernent des GN vides :

. Philippe menace Xavière de $\underline{\underline{\varepsilon}}$ compromettre Jean

Philippe et Jean menacent Xavière de se compromettre l'un l'autre [fusion $\mathrm{x}, \mathrm{z}$ ]

Philippe et Xavière se menacent l'un l'autre de compromettre Jean [fusion $\mathrm{x}, \mathrm{y}$ ]

*Philippe menace Xavière et Jean de (se) compromettre l'un l'autre [fusion y, z]

Ce dernier cas est exclu parce que les arguments fusionnés appartiennent à des prédicats différents.

2.1.2. Verbe source non pronominal, dynamique et GN datif. Intersection du sujet et du datif

GNi V $\alpha$ à $G N j \beta \rightarrow G_{\text {pluri }}$ se $V \alpha\left(\varepsilon_{R} /\right.$ Loc_R $\left.R_{\grave{a}}\right) \beta$

Pour les humains, le GP = à GN peut être pronominalisé par le clitique préverbal lui et indique le destinataire ou bénéficiaire de l'action.

- Max reprochait à Paul son aveuglement nationaliste/Max lui reprochait son aveuglement nationaliste 
Ils se reprochaient $\left(\varepsilon_{\mathrm{R}} /\right.$ mutuellement/les uns aux autres $)$ leur aveuglement nationaliste

Certains verbes pronominaux rentrent aussi dans des structures sources de type: GNi se V à GNj. Dans ce cas, le destinataire GNj n'est pas pronominalisable par un clitique préverbal mais par une forme pleine qui reste sur place et la construction réciproque ne se fait pas en introduisant un nouveau clitique se préverbal :

. Le nucléotide A s'associe au nucléotide T/le nucléotide A s'associe à lui

Les nucléotides s'associent les uns aux autres

Plutôt que d'introduire une règle de redondance se se $\rightarrow$ se, nous préférons traiter ce cas directement (en 2.2). Les prédicats adjectivaux ne peuvent se construire avec des clitiques et seront donc traités aussi en section 2.2.

La forme faible R peut posséder aussi la valeur combinée réfléchie-réciproque :

. i a menti à j

Ils se sont menti ( $\varepsilon_{\mathrm{R}}$ réfl $\vee$ récip/à eux-mêmes/les uns aux autres)

Cette classe est très étendue. Toutes les structures trouvées dans les tables du LADL sont productives. A côté de GNi V à GNj (mentir à, obéir à, parler à, répondre à, sourire à, téléphoner à...), on trouve GNi V GN à GNj : accorder une faveur à, administrer une claque à, avancer du fric à...

Paul a offert un cadeau à Paul

Ils se sont offert des cadeaux $\left(\varepsilon_{\mathrm{R}} /=\right.$ les uns aux autres)

GNi V de GN à GNj : parler de son projet à, rendre compte de sa mission à...

GNi V à GNj de GV : permettre, reprocher...

- Max reproche à Hector de s'agiter pour rien

Ils se reprochent $\left(\varepsilon_{\mathrm{R}} /\right.$ mutuellement / l'un l'autre) de s'agiter pour rien

2.1.3. Verbe source non pronominal, dynamique, avec intersection sujetcomplément adnominal

GNi V Pr $\left[\right.$ Det $N \alpha$ de GNj]GN $\omega \rightarrow G_{\text {pluri }}$ se V Pr Det $N \alpha\left(\varepsilon_{R} /\right.$ Loc_R $\left.\mathbf{R}_{\varepsilon}\right) \omega$

avec $\operatorname{Pr} \rightarrow \varepsilon /$ à / de, le complément $\mathbf{N}$ est une partie du corps ou un objet possédé.

La fusion homogène du sujet et du complément adnominal du second argument ou du locatif se fait très régulièrement lorsque $\mathrm{N}$ est une partie du corps :

. Max serra la main de Paul, Max lui serra la main

Ils se sont serré la main $\left(\varepsilon_{\mathrm{R}} /=\right.$ l'un l'autre)

. Max sauta à la gorge de Paul

Ils se sautèrent à la gorge

- Max arrache les lettres des mains de Sylvie [la variable y est dans le locatif]

Ils s'arrachent les lettres des mains 
Ces formes sont régulières et productives. Par contre avec un objet $\mathrm{N}$ aliénable, il faut introduire un possessif :

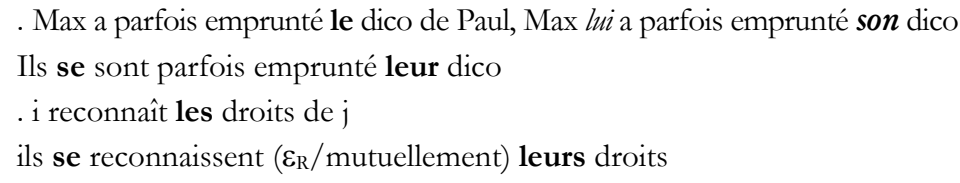

Ces formes sont productives dans la mesure où on peut cliticiser le complément adnominal (Max lui a emprunté son dico). Dans le cas contraire, comme dans
. Max a vu la dernière voiture de Paul
*Max lui a vu sa dernière voiture/Max a vu sa dernière voiture, à Paul
*Ils se sont vu leur dernière voiture/ ?*ils ont vu leur dernière voiture l'un de l'autre

la non pronominalisation par un clitique est corrélée à l'absence de construction réciproque.

\subsection{Constructions réciproques, avec formes faibles non marquées}

Dans cette classe, la forme réciproque faible $\mathrm{GN}_{\text {pluri }} \mathrm{V}$ est non marquée car le prédicat binaire $\mathrm{V}$ n'ayant pas d'emploi absolu (distributif) ne risque pas de produire une ambiguïté. La forme forte est marquée par une locution Loc_R $R_{\operatorname{Pr}}$ $\rightarrow$ ?*mutuellement / entre eux / les uns Pr les autres.

\subsubsection{Verbe symétrique : $\mathrm{GNi} \mathrm{V}$ avec $\mathrm{GNj} \rightarrow \mathrm{GN}_{\text {pluri }} \mathrm{V}\left(\varepsilon_{\mathrm{R}} / \mathrm{Loc} \_\mathrm{R}_{\text {avec }}\right)$}

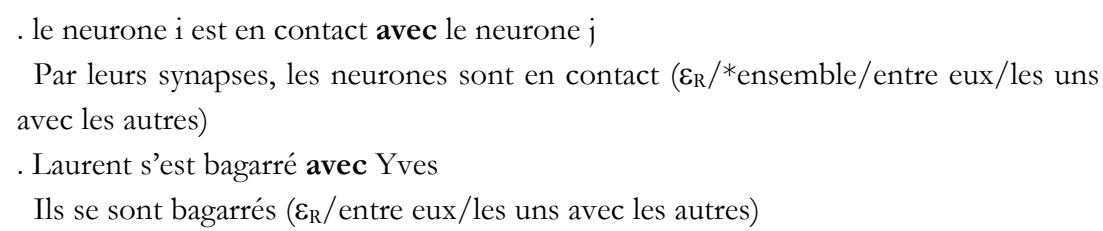

Dans toute cette section, les formes réciproques faibles, comme les neurones sont en contact, ne reçoivent aucune marque de réciprocité ni pronominale ni locutionnelle. Cette structure syntaxique est appelée construction symétrique (par ex. A. Borillo 1971, Boons, Guillet et Leclère 1976). L'étude détaillée de tous les prédicats P rentrant dans cette construction syntaxique montrent qu'ils sont tous « référentiellement» symétriques (ou quasi-symétriques, une petite différence d'agentivité étant possible) et non réflexifs. Leur représentation sémantique satisfait donc $\forall$ xy (P.x.y $\leftrightarrow$ P.y.x) et $\forall \mathrm{x}$ $\neg$ P.x.x: Laurent s'est bagarré avec Yves implique qu'Yves s'est bagarré avec Laurent et l'impossibilité de Laurent s'est bagarré avec lui-même fait que la forme faible n'a pas de valeur réfléchie. Les constructions symétriques ont donc toujours des formes faibles et fortes équivalentes (à un degré d’insistance près). La seule limitation se rencontrant dans des exemples comme : 
. Les labos de génie génétique sont en concurrence

Ce labo est en concurrence avec lui-même

où la valeur réfléchie ne peut se comprendre que dans la mesure où on peut faire éclater le labo en ses parties constitutives rentrant en concurrence.

Toutes les structures $\mathrm{GNi} \mathrm{V}$ avec $\mathrm{GNj}$ ne donnent pas de construction réciproque faible. On peut comparer le prédicat symétrique suivant :

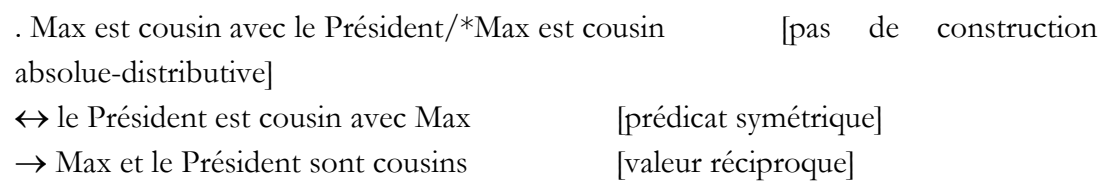
avec un non symétrique tel que être méprisant

$\begin{array}{ll}\text {. Alain est méprisant avec Paul / Alain est méprisant } & \text { [forme distributive] } \\ \rightarrow \text { Paul est méprisant avec Alain } & \text { [prédicat asymétrique] } \\ \text { Ces chercheurs sont méprisants } & \text { [non réciproque] }\end{array}$

Cette dernière forme n'a pas d'interprétation réciproque faible (ils sont hautains vis-à-vis de tout le monde). Une locution forte de réciprocité, telle que les uns avec les autres ou entre eux, est nécessaire pour obtenir l'interprétation réciproque. Ces structures sans forme faible réciproque sont traitées section 3 .

2.2.2. V source : adjectifs ou verbes pronominaux. Intersection sujetobjet.

GNi (cop Adj / se V) (à / de) GNj $\rightarrow$ GN $_{\text {pluri }}\left(\operatorname{cop} \operatorname{Adj} /\right.$ se V) $\left(\varepsilon_{R} /\right.$ Loc_R $\left.\mathbf{R}_{\mathbf{P r}}\right)$

- Ce procédé est analogue à cet autre procédé

Ces procédés sont analogues $\left(\varepsilon_{\mathrm{R}} /=\right.$ les uns aux autres $)$

- Cette approche est complémentaire de celle-là

Ces approches sont complémentaires $\left(\varepsilon_{\mathrm{R}} /=\right.$ les unes des autres $)$

La forme ces procédés sont analogues peut recevoir une interprétation réciproque totalement hors contexte et reste donc non distributive dans cette situation : la forme \#ce procédé est analogue ne s'interprète qu'avec un contexte permettant de récupérer l'ellipse.

Fait remarquable, tous les prédicats adjectivaux que nous avons trouvés dans cette classe sont symétriques! Si le procédé i est analogue au procédé j alors le procédé $\mathrm{j}$ est analogue au procédé $\mathrm{i}$.

Autres ex. : être adjacent à, égal à, lié à, ... ; être contemporain de, complémentaire de, différent de, éloigné de, proche de...

On doit donc en conclure que la construction symétrique ne concerne pas seulement les prédicats symétriques construits avec le GP $\rightarrow$ avec GN, mais aussi les prédicats adjectivaux symétriques construits avec les GP $\rightarrow$ (à / de) GN. 
Comme nous l'avons vu, lorsqu'on part d'un prédicat source déjà pronominalisé, la construction réciproque n'introduit pas de clitique supplémentaire :

. Ce témoignage s'oppose à cet autre

Ces témoignages s'opposent $\left(\varepsilon_{\mathrm{R}} /=\right.$ les uns aux autres/entre-eux)

. Le neurone bipolaire se distingue du neurone multipolaire par sa forme et sa fonction

Les neurones se distinguent $\left(\varepsilon_{\mathrm{R}} /=\right.$ les uns des autres) par leur forme et leur fonction

Dans ces phrases le clitique se est formé sur une relation de neutralité à partir d'une structure du type : Max oppose ce témoignage à celui-là, Max distingue ce neurone de celui-là.

Dans un grand nombre de cas, la préposition avec est aussi possible :

. Les nucléotides A s'associent facilement (aux/avec les) nucléotides T

Les nucléotides A et $\mathrm{T}$ s'associent facilement $\left(\varepsilon_{\mathrm{R}} /\right.$ entre eux/les uns aux autres)

Autres ex. : se confronter à/avec, se fiancer à/avec, s'amalgamer à/avec...

En ce qui concerne la préposition de, la commutation se fait avec d'avec:

. Mon chemin s'est écarté (du/d'avec le) sien

Nos chemins se sont écartés

Autres ex. : se différencier de/d'avec, se séparer de/d'avec...

Il existe aussi des cas d'alternance de/d'avec pour des verbes non pronominaux (qu'il faudrait classer en $\$ 2.1$ ) :

. Mon opinion diffère (de/d'avec) la sienne

Nos opinions diffèrent $(\varepsilon /=$ les une des autres)

2.2.3. Fusion du sujet et d'un complément adnominal, pour des prédicats adjectivaux :

GNi cop Adj Pr $\left[\right.$ Det $\mathbf{N} \alpha$ de GNj] GN $\omega \rightarrow \mathrm{GN}_{\text {pluri }} \operatorname{cop}\left(\varepsilon_{\mathrm{R}} / \mathrm{Loc}_{-} \mathbf{R}_{\varepsilon}\right)$

Adj Pr Poss $N \alpha \omega$

avec $\operatorname{Pr} \rightarrow \varepsilon /$ à / de, et $\mathbf{N}$ partie du corps ou objet possédé. Le Det se transforme en possessif Poss.

- Anne est envieuse (du corsage/des jambes) de Sylvie

Elles sont mutuellement envieuses de (leur corsage/leurs jambes)

- Max est content de l'hospitalité de Paul

Ils sont ( $\varepsilon$ /mutuellement) contents de leur hospitalité

Nous n'avons pas trouvé dans cette classe de verbes sources pronominaux :

- Paul s'oppose à la mère de Sylvie

*Ils s'opposent ( $\varepsilon$ /mutuellement) à (la/leur) mère

ou bien ils n'ont pas de forme faible réciproque (et seront traités section 3) :

. Anne se jette dans les bras de Paul

Ils se jettent dans les bras (*E/l'un de l'autre) 


\subsection{Constructions réciproques fortes, sans forme réciproque faible}

Ces structures n'ont pas de formes réciproques faibles car soit la construction sans locution Loc_R n'existe pas soit elle est distributive (en raison d'un interprétation absolue). Les locutions réciproques mutuellement et entre-eux qui caractérisaient respectivement les classes 1 et 2 ne modifient pratiquement jamais les prédicats de cette troisième classe.

2.3.1. Prédicat source binaire (à 2 arguments nominaux) à construction absolue, distributive

GNi V PrGNj $\rightarrow$ GN $_{\text {pluri }}$ V Loc_R $R_{\operatorname{Pr}}$ avec $\operatorname{Pr} \rightarrow$ à / de / pour / envers...

Il existe une construction absolue : GNmono $\mathrm{V}$

La forme $G_{\text {pluri }} V$ est possible avec une valeur absolue mais pas d'interprétation réciproque faible :

. Max est ingrat à l'égard de Paul

Ils sont ingrats ( $\varepsilon$ distr/ $\neq$ les uns à l'égard des autres)

Max pense à Sylvie

Ils pensent $(\varepsilon / \neq$ les uns aux autres)

Il n'y a pas de forme réciproque faible car la forme Ils sont ingrats construite avec un prédicat distributif (Max est ingrat) implique qu'ils peuvent être ingrat à l'égard de n'importe qui et pas seulement entre-eux.

Autres ex. : être amoureux de, content de..., être antipatbique à, indifférent à..., être bon pour, affable envers..., faire attention à, s'babituer à, penser à, résister à..., s'éloigner de...

\subsubsection{Prédicat source binaire, sans construction absolue}

\section{GNi V Pr GNj $\rightarrow$ GN $_{\text {pluri }}$ V Loc_R $\mathbf{R}_{\mathbf{P r}} \quad$ avec $\operatorname{Pr} \rightarrow$ à / de / sur / Loc}

Il n'existe pas de construction $\mathrm{GN}_{\text {mono }} \mathrm{V}$. La forme $\mathrm{GN}_{\text {pluri }} \mathrm{V}$ n'existe pas non plus (contrairement aux classes 1.2, 2.2 et 3.1) et il n'y a donc pas d'interprétation réciproque $\mathrm{R}$ faible

. i s'adresse à j

Pour le savoir, ils s'adressent ( ${ }^{*} \varepsilon /$ les uns aux autres)

- ma décision dépend de la sienne

Nos décisions dépendent (*E/les unes des autres)

. i s'effondre sur $j$

S'il n'y avait que des forces attractives, les atomes s'effondreraient $\left({ }^{*} \varepsilon /\right.$ les uns sur les autres)

. Max entra et alla droit à Sophie.

Ils entrèrent et allèrent droit $\left({ }^{*} \varepsilon /\right.$ l'un à l'autre)

On pourrait classer les comparatifs dans cette catégorie :

. $\mathrm{i}$ est (aussi/plus) bête que $\mathrm{j}$

Ils sont (aussi/plus) bêtes $\left({ }^{*} \varepsilon /\right.$ les uns que les autres) 
bien que l'interprétation résultante ait plus une valeur intensive que réciproque.

\subsubsection{Verbe distributif, avec fusion du sujet et d'un spatio-temporel \\ GNi GV Loc GNj $\rightarrow$ GN $_{\text {pluri }}$ GV Loc_R $\mathbf{R}_{\text {Pr }}$}

GN GV est distributif, avec Loc $\rightarrow$ devant / sur / vers...après

La forme $\mathrm{GN}_{\text {pluri }} \mathrm{GV}$ a une interprétation distributive mais pas d'interprétation réfléchie-réciproque faible. Elle peut être construite sur un verbe intransitif :

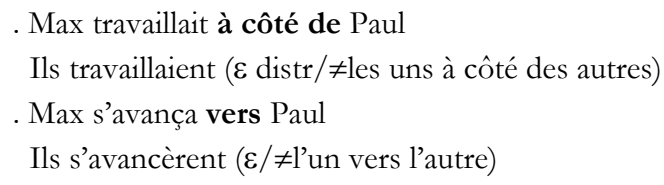

ou verbe binaire (transitif direct ou indirect)

. Max regardait le film à côté de Fabienne

Ils regardaient le film $(\varepsilon / \neq 1$ 'un à côté de l'autre)

En ce qui concerne les expressions temporelles, on observe une claire dissymétrie entre les trois expressions temporelles :

- Max sorti (avant/après/en même temps que) Paul

Les étudiants sortirent ( $\varepsilon /$ les uns ( ?avant/après/*en même t..) les autres)

\subsection{Construction d'une classe par fusion des objets direct et indirect GN V GNi Pr GNj $\rightarrow$ GN V GN pluri $_{\text {AdvR }}$ Pr}

- Max a versé le rhum dans le cognac

Max a versé les alcools ( ${ }^{*} \varepsilon /$ les uns dans les autres)

Avec la préposition avec des objets symétriques, on retrouve une forme réciproque faible qu'il faudrait traiter section 2 :

. Max a mélangé le rhum (avec/et) le jus

Max a mélangé les liquides $\left(\varepsilon_{\mathrm{R}} /=\right.$ les uns avec les autres)

Expression temporelle :

- Max embrassa Sylvie (avant/après/en même temps que) Sophie

Max embrassa les étudiantes (les unes (*avant/après/*en...) les autres)

\section{Conclusion}

Ni distributive ni collective, la quantification réciproque de type $<1,2>$, peut se comprendre comme une accumulation itérative des extensions de deux référents disjoints, liés par un prédicat (locatif inclus). Nous avons tenté de définir des critères syntactico-sémantiques, permettant de rassembler l'ensemble des formes réciproques dans des classes relativement homogènes. Les nombreuses restrictions tant syntaxiques que sémantiques mises en évidence dans ce travail laisse espérer qu'une 
partie importante des contraintes portant sur la représentation logique puisse en être tirée sans devoir faire un recours massif au contexte.

Francis RENAUD

EHESS, CRLAO

54 Bd Raspail

75006 PARIS

Francois.Renaud@ehess.fr

\section{ANNEXE}

Considérons quelques exemples typiques d'interprétation réciproques :

(1) Les citoyens sont tous égaux de droit (les uns aux autres)

Glose : N'importe quel citoyen est égal de droit à n’importe quel autre citoyen.

$\forall \mathrm{x} \forall \mathrm{y}(($ citoyen. $\mathrm{x} \wedge$ citoyen.y $\wedge \mathrm{x} \neq \mathrm{y}) \rightarrow$ égal-de-droit.x.y $)$

(2) Les vertèbres s'articulent toutes les unes aux autres vertèbre

Glose: N'importe quelle vertèbre s'articule à (*n'importe quelle/une) autre

$\forall \mathrm{x}($ vertèbre. $\mathrm{x} \rightarrow \exists \mathrm{y}($ vertèbre.y $\wedge \mathrm{x} \neq \mathrm{y} \wedge$ s-articuler.x.y $))$

(3) Les documents étaient posés en désordre les uns sur les autres

Glose: N'importe quel document, était posé en désordre en dessus ou en dessous un ou plusieurs autres documents (l'alternance posé en dessous doit être rajoutée pour les documents complètement en dessous).

$\forall \mathrm{x}($ document. $\mathrm{x} \rightarrow \exists \mathrm{y}($ document.y $\wedge \mathrm{x} \neq \mathrm{y} \wedge$ (posé-désordre.x.(sur.y) $\vee$ posédésordre.y.(sur.x))))

Peut-on trouver une valeur générale, valable dans tous les cas. On pourrait être tenté de garder la formule la plus générale (celle de l'exemple 3), mais appliquée à (1), elle donne une interprétation difficile à accepter : pour n'importe quel citoyen, il existe un ou plusieurs autres citoyens auxquels il est égal.

\section{BIBLIOGRAPHIE}

BOONS, J.-P., A. GUILLET, et al., 1976, La structure des phrases simples en français. Constructions intransitives, Droz, Genève.

BORILLO, A., 1971, «Remarques sur les verbes symétriques français », Langue Française, 11, pp. 17-31. 
Francis Renaud

DALRYMPLE, M., M. KANAZAWA, et al., 1998, "Reciprocal Expressions and the Concept of Reciprocity", Linguistics and Philosophy, 21/2, pp. 159-210.

GUILLET, A. and C. LECLÈRE, 1992, La structure des phrases simples en français, Librairie DROZ, Genève.

HEIM, I., H. LASNIK, et al., 1991, « Reciprocity and Plurality », Linguistic Inquiry, 22/1, pp. 63101.

KEENAN, E., 1987, «Unreducible n-ary Quantifiers in Natural Language », in P. Gardenfors, Generalized Quantifiers : Linguistics and Logical Approaches, D. Reidel, Dordrecht.

SCHNEDECKER, C. and M. BIANCO, 2000, AVEC entre conjonctif et comitatif : aspects linguistiques et psycholinguistiques. Colloque Tel Aviv.

WESTERSTAHL, D., 1994, "Quantifiers in Formal and Natural Languages », in D. Gabbay and F. Geuntner, Handbook of Philosophical Logic, Reidel, Dordrecht, 4. 